Josef Burri

\section{Einleitung}

Die Traditionelle Thailändische Medizin (TTM) und damit auch die Lehre von der traditionell richtigen Ernährung beruhen auf der Lehre von den vier Kernelementen (thaat). (Die Traditionelle Chinesische Medizin und die Ayurveda-Medizin unterscheiden hingegen fünf Elemente [1, 2].) Im Folgenden soll dargestellt werden, wie sich aus der Lehre von den vier Kernelementen die gesundheitlichen Wirkungen der thailändischen Ernährung ableiten. Selbstverständlich handelt es sich dabei nicht um kurative Medizin, sondern um ein System tradierter Erfahrungswerte, wie die Gesundheit des Menschen durch eine massvolle, ausgeglichene und individuellen Kernelementen angepasste Ernährung gefördert werden kann [3].

Krankheiten entstehen nach dem Verständnis der TTM durch ein Ungleichgewicht der Elemente im einzelnen Menschen. Mit fortschreitendem Alter verlieren die Elemente ihre Energie. Mit dem Tod ist die Lebenskraft der Elemente zum Erliegen gekommen. Das Ziel der Ernährung ist nicht etwa, dass nur die dem Kernelement zugeordneten Nahrungsmittel verwendet werden dürfen. Vielmehr soll ein harmonischer Ausgleich zwischen den Elementen geschaffen werden, wobei sich höchstens die Schwerpunkte gemäss der Elemente-Lehre verändern. Ausserdem verschaffen auch «neutrale» Nahrungsmittel wie Reis sowie Fisch- und Hühnerbrühe eine Verbindung zwischen den unter-

\title{
Gesund bleiben mit der thailändischen Küche - eine Ernährung im Einklang mit den Elementen
}

Seit über 30 Jahren erfreuen sich Thai-Restaurants hierzulande grosser Beliebtheit. Das Thai-Essen ist inzwischen in Europa äusserst populär und gilt als schmackhaft, bekömmlich, gesund und manchmal auch als etwas scharf. Über die Küche dringen thailändische Wörter in westliche Sprachräume ein. Asiatische Restaurants tragen mitunter fantasievolle Namen: «Pet Noy Thai Takeaway» («Etwas scharfer Thai-Takeaway»), «Ban Thai» und «Rüan Thai» («ThaiHaus»), «Chok Dee» («Viel Glück») und dergleichen. Auch die «Tom Khaa Kai» (Hühnersuppe mit Kokosmilch) und die «Tom Jam Kung» (scharfsaure Suppe mit Garnelen) findet Eingang in die Ess- und Genussgewohnheiten ausserhalb der thailändischen Landesgrenzen. In Thailand wird bei aller «Verwestlichung» an den traditionellen Speisen festgehalten - sie gelten als besser verträglich und gesünder als andere Kostformen und sind Teil des kulturellen Selbstverständnisses in den (etwas vereinfacht gesagt) vier Landesteilen. Es handelt sich um eine variantenreiche Küche, die tages- und jahreszeitliche Faktoren ebenso berücksichtigt wie individuelle Voraussetzungen. Der nachfolgende Beitrag versucht zu klären, was die gesundheitsfördernde Wirkung der thailändischen Ernährung ausmacht und wie sie angewendet werden sollte, damit sie die erwünschten Effekte erzielt.

schiedlichen Kräften, die den Nahrungsmitteln innewohnen. Die richtige Balance ist entscheidend.

Jede Person verfügt über ein eigenes Kern- oder Heimelement, das die anderen drei Elemente dominiert. Es wird durch das Datum der Empfängnis und der Geburt bestimmt. Die Kernelemente tragen einen Namen, der ihre Eigenschaften charakterisiert.

- Erde: Das Element Erde macht die festen Bestandteile des Körpers aus, nämlich Haar, Nägel, Zähne, Körperhaut, Muskeln, Sehnen, Bänder, Knochen, Knochenmark, Milz, Herz, Leber, Nieren, Lunge, Magen, Darm und Gehirn.

- Wasser: Das Element Wasser besteht aus den flüssigen Bestandteilen, die für das Überleben wichtig sind, nämlich Gallenflüssigkeit, Schleim, Lymphflüssigkeit, Blut, Schweiss, Fett, Tränen, Speichel, Gelenkflüssigkeit und Urin.

- Wind (oder Luft): Das Element Wind ist durch die Bewegung innerhalb und ausserhalb des Körpers charakterisiert, also durch die Blutzirkulation, den Atem und die Verdauung.

- Feuer: Das Element Feuer manifestiert sich durch seine Hitze, die den Körper warm hält und damit die Energien für sein Funktionieren freisetzt, ihn aber auch verbrennt und zerstört.

Für die Bestimmung des Kernelementes ist ein Experte für TTM erforderlich, wobei er nicht nur den Geburtsort und das exakte Geburtsda-

\section{KARGER}

(C) 2016 S. Karger GmbH, Freiburg

Fax +497614520714 
tum, sondern auch die Länge der Schwangerschaft der Mutter wissen will. Mit etwas Glück kann man eine Bestimmungsscheibe erwerben, mit der das Kernelement errechnet werden kann [4]. Die Kenntnis des eigenen Kernelementes ist eine Voraussetzung für die individuell zuträgliche Ernährung. Allerdings kann sich das Kernelement im Verlauf des Lebens auch verändern. Einige Menschen vereinen in sich zwei Kernelemente. Stehen weder Bestimmungsscheibe noch kompetente Heiler zur Verfügung, setzt die Selbstreflexion zur möglichen Bestimmung des Kernelementes ein und löst möglicherweise einen individuellen Bewusstseinsprozess in Richtung einer zuträglichen Ernährung aus.

\section{Die Kernelemente und die entsprechende Ernährung}

\section{Erde}

Das Element steht symbolisch für die festen Strukturen des menschlichen Körpers und für das tragende geistige Fundament. Eigentlich sind Erde-Menschen sehr treu und stabil in den Beziehungen; manchmal ist aber auch das Gegenteil zutreffend. Der Grund dafür liegt in ihrer Empfindlichkeit gegenüber äusseren Einflüssen. Emotionale Verletzungen und Partnerverlust hinterlassen tiefe Narben. Auch nasskaltes Wetter kann Schmerzen im Bereich von Muskeln, Sehnen und Gelenken verursachen. Leicht treten Erkältungen auf.

Ballaststoffe und aromatische Gewürze wie Muskatnuss, Sternanis und Zimt stimulieren die träge Verdauung. Dies gilt vor allem für kalte Wintertage. Übergewicht kann ein Problem sein. Erde-Menschen benötigen Nahrungsmittel mit zusammenziehenden, butterartigen (öligen), süssen und salzigen Eigenschaften. Alle Arten von Bohnen sowie Linsen und Nüsse unterstützen die gesundheitliche Stabilität. Haut und Haare brauchen viel Pflege und Feuchtigkeit. Es ist auf ge- nügend Flüssigkeitszufuhr zu achten. Ein guter und tiefer Schlaf verbessert die ohnehin exzellente Leistungsfähigkeit von Erde-Menschen.

\section{Wasser}

Wasser-Menschen lieben andere Menschen und haben ein starkes Mitgefühl für alle Kreaturen. Sie sind grosszügig und verfügen über ein exzellentes Gedächtnis. Hinter einer liebenswürdigen Fassade kann sich aber auch Verletzlichkeit verstecken, und schlimmstenfalls kommen sie sich verloren und ausgegrenzt vor. Eine Person mit dem Wasser-Element liebt warme Temperaturen und leidet unter kaltem Regenwetter. Wie immer kann auch das genaue Gegenteil der Fall sein. Fühlt sich jemand bei kalten Regenschauern wohl, dann ist das ein sehr auffälliges Symptom, das auf Wasser als Kernelement schliessen lässt. Normalerweise braucht ein Wasser-Mensch viel Schlaf, um sich wieder zu erholen. Er muss genügend trinken, um Atmung, Verdauung, Ausscheidung und Sexualität in Gang zu halten. Eine Störung des Gleichgewichts der Körperfunktionen führt zu Erkältungen, Bronchitis, Allergien und Verdauungsbeschwerden.

Fetthaltige und schwere Mahlzeiten passen nicht zu Wasser-Menschen, Bitteres und Saures hingegen schon, vor allem bei kaltem Wetter. Auch eher fad schmeckende Früchte und Gemüse wie Okra, Sprossen, Pilze und Aubergine sind bekömmlich für sie.

\section{Wind}

Wind-Menschen sprühen vor Kreativität und Unternehmungslust. Deshalb essen sie, um zu überleben, aber sie leben nicht, um zu essen. Demzufolge sind sie nicht wählerisch, sondern schnappen sich hier und da, was gerade erhältlich ist. Natürlich ist das ungesund, nicht nur für Wind-Menschen, aber für sie besonders, da ihre Verdauung schnell einmal rebelliert. Daher sollten sie sich regelmässige
Mahlzeiten angewöhnen und die für sie passenden Speisen sorgfältig aussuchen. Weitere Schwachstellen, vor allem bei feuchtkaltem Wetter, sind steife Knochen und Gelenke sowie der Atemapparat. Die Blutzirkulation kann träge sein, und rasch stellen sich Erschöpfung und Erkältungen ein. Wind-Menschen können aber auch psychisch ganz schön durcheinandergewirbelt werden - nach traditioneller Vorstellung unter dem schädlichen Einfluss von bösen Geistern [5].

Weniger geeignet sind rotes Fleisch und Meeresfrüchte sowie blähende Gemüse wie alle Kohlarten. WindMenschen vertragen hingegen Wurzelgemüse im Allgemeinen gut. Windtreibende Gewürze wie Kümmel, Kardamom, Minze und Ingwer, aber auch scharfe Gewürze wie Chili helfen bei der Verdauung und wärmen den Körper, vor allem bei klimatischer Kälte. Allzu Saures sollten sie meiden; Getränke mit natürlicher Süsse wie Karottensaft sind bekömmlich für sie.

\section{Feuer}

Das Feuer verwandelt die aufgenommene Nahrung in Energie für Körper und Geist. Menschen mit dem Element Feuer vertragen die Hitze nicht gut oder aber sie frösteln ständig und ziehen sich selbst im Sommer warm an. In der Tat neigen sie bei kühlem und nassem Wetter zu Erkältungen. Sie müssen sich auch um ihre Haut sorgen, da sie leicht austrocknet und ständiger Pflege bedarf. Ihre Verdauung neigt zu Überreaktionen; in der Folge stellen sich beispielsweise saures Aufstossen und Magengeschwüre ein. Charakterlich sind Feuer-Menschen sehr selbstbewusst, energisch und zielstrebig. Sie kommen mit wenig Schlaf aus und sind ständig aufTrab. Geduld und Einfühlungsvermögen gehören nicht zu ihren Stärken. Sie haben etwas Zerstörerisches an sich, wenn sie die Beherrschung verlieren; sie können aber kritische Situationen mit ihrer Geradlinigkeit zielsicher meistern. Menschen mit 
dem Element Feuer haben einen unersättlichen Appetit. Sie müssen also vorerst ihren Appetit zügeln und ihre Nahrung anpassen, damit sie nicht unter Sodbrennen, Übergewicht, Verstopfung oder Diabetes leiden.

Ihre Hauptmahlzeit ist eindeutig am Mittag, der durch das Feuer-Element bestimmt wird. Rotes Fleisch, Alkohol und Kaffee fördern die innere «Hitze» und sollten deshalb von Feuer-Menschen eher gemieden werden. Passend sind hingegen Nahrungsmittel mit faden, bitteren und kühlenden Eigenschaften wie Bohnensprossen, Pilze sowie Chinakohl.

\section{Berücksichtigung von Tageszeit, Jahreszeit und Klima}

Jedoch auch die Tageszeiten und das Klima, also die äussere Welt, sind durch ein Kernelement charakterisiert und können durch eine angepasste Wahl der Lebensmittel beeinflusst werden:

- 6-10 Uhr: Element Wasser;

- 10-14 Uhr: Element Feuer;

- 14-18 Uhr: Element Wind;

- 18-22 Uhr: Element Wasser;

- 22-2 Uhr: Element Feuer;

- 2-6 Uhr: Element Wind.

Ein gesundes Frühstück sowie das Abendessen bestehen somit aus Nahrungsmitteln des Elementes Wasser und des Kernelements, während das Mittagessen das Element Feuer und das Kernelement berücksichtigt.

Thailand kennt drei Jahreszeiten, in denen ein bestimmtes Kernelement vorherrscht. Wir haben das Klimasystem den geografischen Zonen mit vier Jahreszeiten angepasst:

- Nasskalt: Element Erde;

- Kühl und trocken: Element Wasser;

- Nass und eher warm: Element Wind;

- Heiss: Element Feuer.

Bei nasskaltem Wetter fügt die traditionsbewusste thailändische Hausfrau dem Speiseplan Nahrungsmittel des Elementes Erde bei, z.B. Ingwer, Kardamom, Asiatischer Basilikum

Kasten. Wald-Curry (Kähng Paa)

Kähng Paa (wörtlich: Wald-Curry) ist eine typische Jahreszeiten-Suppe Thailands. Sie existiert in zahlreichen geografischen Varianten und enthält je nach klimatischen Gegebenheiten sehr unterschiedliche Zutaten - im Nordosten manchmal Froschfleisch, im Norden Wildschwein und am Meer Fisch. Wichtiger als die tierischen Ingredienzen sind jedoch die Gewürze und das jeweilige saisonale Gemüse. Wegen ihrer Schärfe wird das Wald-Curry in der kalten Jahreszeit geschätzt, weil es den Körper erwärmt, und in der heissen Jahreszeit, weil es den Körper zum Schwitzen und damit zum Abkühlen bringt. Kähng Paa kommt also vor allem den Bedürfnissen der Menschen mit den Kernelementen Wind und Feuer entgegen. Das folgende Rezept ist nur eine von vielen Möglichkeiten, wie ein schmackhaftes Wald-Curry hergestellt werden kann.

Zutaten:

- Hühnerbrühe (kann durch eine Gemüsebrühe ersetzt werden)

- Getrocknete Chili (Phrik Khie Nuh = Capsicum minimum oder Phrik Chie Fah =

Capsicum frutescens)

- Zwiebel, grob gehackt

- Knoblauchzehen, gequetscht

- Galgantwurzel, geschält und in feine Scheiben geschnitten

- Fingerwurz (der Länge nach in feine Streifen geschnitten)

- Zitronengras (nur das Innere verwenden, fein geschnitten)

- Frische grüne Pfefferschoten

- Kleine grüne Auberginen

- Pilze (die Sorte ist nicht festgelegt, in Nordthailand oft Hed lom = Lentinus polychrous, 30 min Kochzeit)

- Meersalz (nach Belieben)

- Palmzucker (nach Belieben)

- Dill oder Thai-Basilikum (nach Belieben)

Zubereitung:

Die Menge der Zutaten ist nicht festgelegt. Man nimmt, was es hat. Manche Thai-Köche verwenden Curry-Paste, Fischsauce, Garnelenpaste (Kapi) oder Fischpaste (Pla rah), um die Suppe abzurunden oder dickflüssiger zu machen. Das ist aber Geschmackssache. Auch beim Gemüse herrscht eine grosse Freiheit. Meist werden aber grüne oder grünblättrige Gemüse verwendet (z.B. die Blätter der Scharlachranke $=$ Tamlüng, Flügelbohnen und anderes). Alle Zutaten werden in einem Topf bei geringer Hitze geköchelt. Falls das Curry nicht als Suppe, sondern als Hauptmahlzeit zu Reis gegessen wird, weniger Flüssigkeit verwenden.

Zuordnung der Elemente:

- Hühnerbrühe: alle

- Chili: Wind

- Zwiebel: Wind, Feuer

- Knoblauch: Wind

- Galgant: Wind

- Fingerwurz: Erde

- Zitronengras: alle

- Pfefferschoten: Wind

- Aubergine: Wasser, Feuer

- Pilze: Erde, Wasser, Feuer

- Dill: Wind, Feuer

- Basilikum: Wind, Feuer

(Ocimum canum, Maenglack). Bei Hitze sind leicht verdauliche Nahrungsmittel wie Früchte und Gemüse empfehlenswert. Kühlende Eigenschaften haben Bittergurke, Kürbis und Gurken generell. Bei den Früchten sind es vor allem Ananas, Rosenapfel, Melonen und Birnen. Fett, Kohlenhydrate und Zucker sollten reduziert werden, während Suppen vorteilhaft sind und dem Körper Flüssigkeit zu- führen. Unter den Heilpflanzen wird der Indische Wassernabel (Centella asiatica, Gotu Kola) empfohlen. Im Kasten findet sich ein Rezept für heisse und kalte Tage.

Der Geschmack (rot) eines Nahrungsmittels ist ebenfalls einem der vier Elemente zugeordnet:

- Element Erde: zusammenziehend (adstringierend), butterartig, salzig, süss; 
- Element Wasser: bitter, mild, sauer;

- Element Wind: scharf, erfrischend (kühl);

- Element Feuer: bitter, mild, erfrischend (kühl).

Eine ausgewogene Ernährung berücksichtigt all diese Aspekte: das individuelle Heim- oder Kernelement, das Klimaelement und das Tageszeitenelement ebenso wie die Geschmacksnuancen. Sie wirkt ausgleichend und fördert die Gesundheit des Menschen. Tabelle 1 ordnet eine Vielzahl von Nahrungsmitteln den einzelnen Elementen zu. Typisch für thailändische Gerichte ist an und für sich immer die Kombination von mehreren, sehr unterschiedlichen Zutaten. So gehören in eine Sauce für thailändische Salate (yam) Fischsauce, $\mathrm{Zu}$ cker, Limettensaft und Chili, also eine Kombination der geschmacklichen Nuancen salzig, süss, sauer und scharf. Fleisch, Fisch und Garnelen sind in der thailändischen Küche durchaus erlaubt und in den meisten Gerichten präsent, allerdings in jeweils geringen Mengen und eher als Beilage zu rohem oder kurz im Wok gebratenem Gemüse.

\section{Verbreitung in Thailand}

Die Lehre von den vier Kernelementen ist in der breiten Bevölkerung und besonders bei der thailändischen Hausfrau und jüngeren Leuten wenig bekannt und bleibt als Theorie den wirklichen Spezialisten im Gesundheitsbereich vorbehalten. Demgegenüber ist aber das praktische Wissen über die gesundheitlichen Eigenschaften einzelner Nahrungsmittel weit verbreitet, beispielsweise ob eine Frucht als «heiss» oder «kühlend» eingeschätzt wird. Die Jüngeren lernen von den Eltern und Grosseltern [6] und zunehmend auch aus Bildungskampagnen, Schulprogrammen und den lokalen Medien, die sich dem Thema Essen und Trinken widmen. So weiss eigentlich schon fast jedes Kind, dass die Durian-Frucht (Durio zibethinus, Thurian) Hitze verursacht. Dummer- weise gelangt diese "Stinkfrucht» in der heissen Jahreszeit (März bis Mai) auf den Markt, weil sie dann reif ist; es wird deshalb angeraten, Durian nie in Kombination mit anderen "heissen» Nahrungsmitteln wie beispielsweise Alkoholika zu konsumieren. Das Konzept von «heissen» und «kalten» Nahrungsmitteln scheint in weiten Teilen Asiens verbreitet und stammt vermutlich aus der Ayurvedischen Medizin [7], die ebenfalls Nahrungsempfehlungen auf der Basis von Konstitutionstypen abgibt.

Manchmal sind auch regionale Varianten zu konstatieren: In Zentralund Südthailand gilt Klebreis (khaaw niau, sticky rice) als «heiss», im Nordosten jedoch als «neutral». Die unterschiedliche Zuordnung hat wohl mit der Zubereitung zu tun; ausserhalb des Nordostens und des Nordens, wo Klebreis zur täglichen Basisnahrung gehört, wird dieser für die Herstellung von schwerer verdaulichen Süssspeisen verwendet und ist dann definitionsgemäss nicht mehr «neutral». Die thailändische Kulinarik ist ein häufig diskutiertes Thema im Familien- und Freundeskreis. Im Berufsalltag ist die Berücksichtigung der individuell, klimatisch und zeitlich zuträglichen Ernährung nur bedingt möglich. Besonders in urbanen Verhältnissen und im aufstrebenden Mittelstand greift eine global verbreitete Wohlstandsernährung sowie Fast-Food-Ernährung mit mehr Fett, Fleisch und Zucker um sich, mit den bekannten negativen Folgen für die Gesundheit wie Übergewicht [8] sowie kardiovaskuläre und zerebrovaskuläre Erkrankungen [9, 10]. Bei den Mahlzeiten im Familienkreis und anlässlich von Festen, wenn Menschen mit unterschiedlichen Kernelementen zusammenkommen, besteht jedoch durchaus eine individuelle Auswahlmöglichkeit, indem sehr unterschiedliche Speisen in grösserer Zahl auf den Tisch kommen und jeder sich nach Belieben bedient.

\section{Thai-Food gegen Krebs?}

Im Zusammenhang mit der thailändischen Ernährung stellt sich die Frage, ob bestimmte Krankheiten wie Krebs durch die thailändische Küche vermieden oder bekämpft werden können. Die Zubereitungen und Empfehlungen der traditionellen Heiler in Thailand beruhen praktisch durchgehend auf der Vier-Elemente-Lehre. Hat eine Ernährung auf dieser Basis nicht nur antioxidative und entzündungshemmende Wirkungen, sondern vermag sie auch aktiv schädliche Zellteilungen zu hemmen? Diese Frage ist umso mehr berechtigt, als Forscher davon ausgehen, dass 30\% der Krebserkrankungen in einem Zusammenhang mit der individuellen Ernährungsweise stehen und dass erhebliche geografische Unterschiede in der Häufigkeit von bestimmten Krebsarten und anderen Krankheiten bestehen $[11,12]$. So hat man beispielsweise festgestellt, dass das Risiko, an bestimmten Krebsarten wie Brust- und Prostatakrebs zu erkranken, bei einer Gruppe von Japanern, die in die USA ausgewandert sind, wegen der Anpassung an die amerikanischen Ernährungsgewohnheiten um den Faktor 10 zugenommen hat.

Die traditionelle thailändische Küche wird ganz allgemein als gesundheitsverträglich eingestuft, und zwar aus folgenden Gründen:

- geringer Fleischkonsum (kleine Menge pro Mahl);

- häufiger Fischkonsum (Zufuhr von wertvollen Omega-3-Fettsäuren);

- viele Früchte und Gemüse;

- therapeutisch wirksame, frische Gewürze;

- hoher Ballaststoffgehalt;

- rasche und schonende Zubereitung;

- hoher Rohkostanteil.

Es ist deshalb nicht überraschend, dass auf dem thailändischen Speiseplan manche Nahrungsmittel zu finden sind, die in der Krebsforschung als Schutz vor Krebs und zur Verlangsamung des Krebswachstums eingesetzt werden. Dazu gehören beispielsweise 


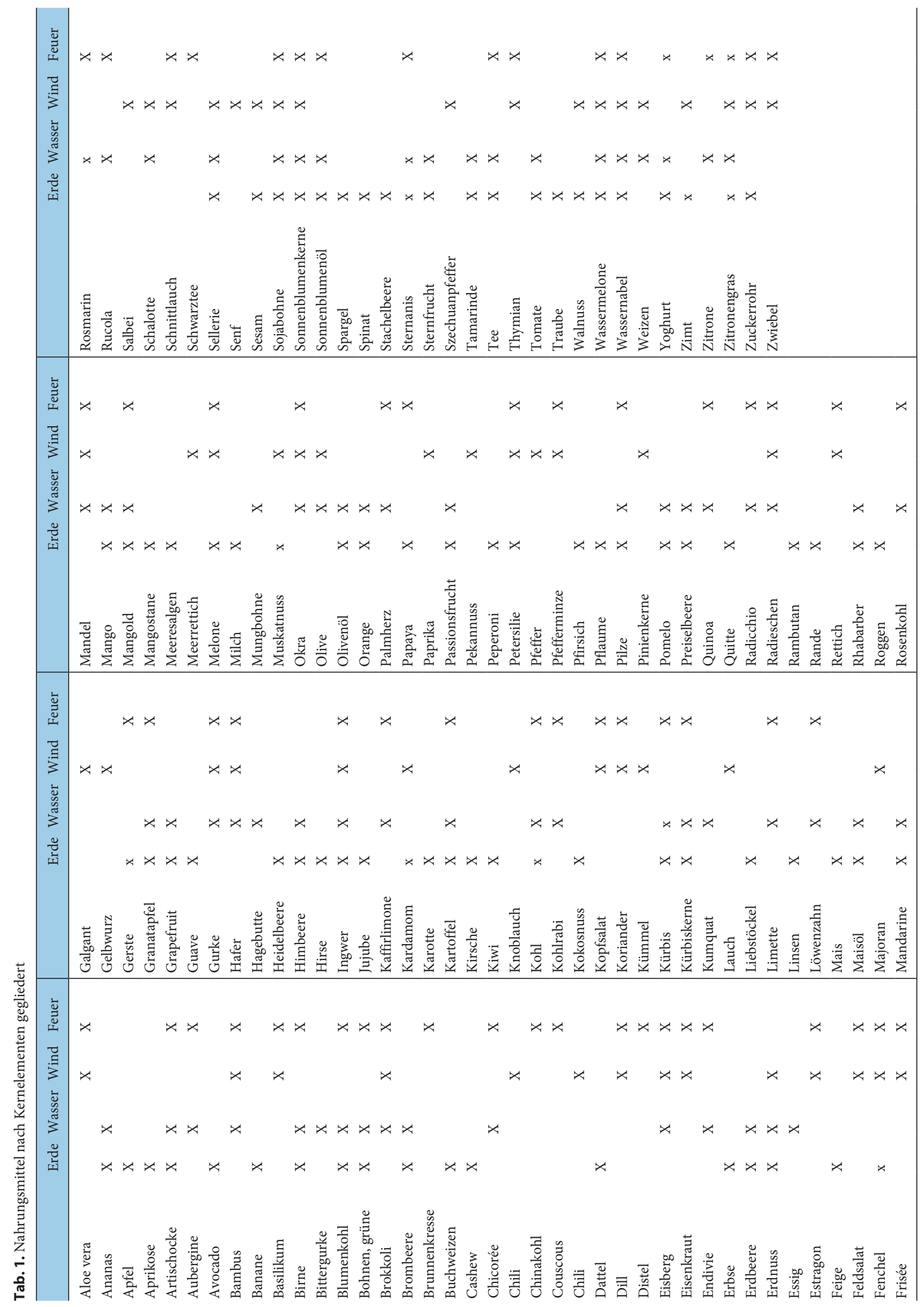


Tomaten (die in Thailand als Garantie für langes Leben betrachtet werden), Produkte aus Soja (Tofu, Sprossen, Mehl), grüne Bohnen, Zwiebeln, Brokkoli, verschiedene Kohlarten, Knoblauch, Ingwer, Mango, Guave, Wassermelone, Papaya, Orange und Sesam [13]. Die TTM kennt eine ganze Reihe von Früchten sowie Gewürz- und Heilpflanzen, denen eine präventive Wirkung gegen Krebserkrankungen zugeschrieben werden: Mangostan, Fingerwurz, Galgant, Gelbwurz, Wassernabel, Pekinggras, Indische Stachelbeere, der Pilz namens Hed lom oder auch Stachelannone, um nur einige zu nennen.

\section{Diskussion und Fazit}

Die thailändische Ernährungslehre von den vier Kernelementen, die vier
Grundtypen zugeordnet sind, ist Teil der TTM. Leider wurde bisher versäumt, die gesundheitliche Relevanz eines solchen differenzierten und individuellen bzw. typenspezifischen Ernährungssystems auf den Prüfstand zu stellen und mit globalisierten Ernährungskonzepten $\mathrm{zu}$ vergleichen. Generell ist anzunehmen, dass die Küche durch die verwendeten Zutaten und Prozeduren als eine weitgehend vegetarische Kostform günstig ist. Die Spezifität bezüglich der traditionellen vier Grundtypen wäre ebenfalls durch klinische Studien zu überprüfen - indem man z.B. bestimmte Patientengruppen mit entsprechenden Grundtypen eine Zeit lang die entsprechend ihrem Grundtyp ausgesuchte Nahrung und dann eine Zeit lang eine sozusagen «falsche» Ernährung mit einem anderen Grundtyp «verabreicht». Dabei wären Kurzzeiteffekte natürlich sehr viel einfacher zu eruieren als Einflüsse auf die langfristige Erhaltung der Gesundheit.

Insgesamt lässt sich immerhin sagen: Eine Ernährung gemäss der thailändischen Küche bietet eine individuell angepasste, ausgewogene und vielfältige Nahrungszufuhr, welche das Wohlbefinden steigert und dazu beträgt, dass Körper und Geist über lange Zeit gesund erhalten bleiben. Neben dem unerlässlichen Blick auf die Krankheit ist der typenerprobte Blick auf den Menschen in seiner Gesamtheit immer wieder eine Herausforderung für Menschen, die im Dienste von Gesunderhaltung und Heilung tätig sind.

\section{Literatur}

1 Chokevivat V, Chutthaputti A: The Role of Thai Traditional Medicine in Health Promotion. 6th Global Conference on Health Promotion. Bangkok, 2005.

2 Burri J: Thailands traditionelle Medizin: Ein Überblick. Schweiz Z Ganzheitsmed 2013;25: 169-179.

3 Chaithavuthi J, Muangsiri K: Ancient Thai Massage - Healing with Life Force. Chiang Mai, Thai Massage Book Press, 2012.

4 Su-Mei Yu: The Elements of Life - a Contemporary Guide to Thai Recipes and Traditions for Healthier Living. Hoboken, John Wiley \& Sons, 2009.

5 Golomb L: An Anthropology of Curing in Multiethnic Thailand. Urbana, IL, University of Illinois Press, 1985, pp 137-138.

6 Aree P, Tanphaichitr V, Suttharangsri W, Kavanagh $\mathrm{K}$ : Eating behaviors of elderly persons with hyperlipidemia in urban Chiang Mai. Nurs Health Sci 2004;6:51-57.

7 Obeyesekere G: The impact of Āyurvedic ideas on the culture and the individual in Sri Lanka; in Leslie C (ed): Asian Medical Systems: A Comparative Study. Berkeley, University of California Press, 1976, pp 201-226.

8 Chavasit V, Kasemsup V, Tontisirin K: Thailand conquered under-nutrition very successfully but has not slowed obesity. Obes Rev 2013;14(suppl 2):96-105.

9 Kosulvat V: The nutrition and health transition in Thailand. Public Health Nutr 2002;5: 183-189.
10 Thailand Health Data 2012-2013. Nonthaburi, Alpha Research, 2013.

11 Béliveau R, Gingras D: Krebszellen mögen keine Himbeeren - Nahrungsmittel gegen Krebs - Das Immunsystem stärken und gezielt vorbeugen. München, Wilhelm Goldmann, 2010.

12 Béliveau R, Gingras D: La méthode anticancer - comment réduire les risques. Paris, Flammarion, 2014.

13 Knasmüller S (Hrsg): Krebs und Ernährung Risiken und Prävention - wissenschaftliche Grundlagen und Ernährungsempfehlungen. Stuttgart, Thieme, 2014. 